\title{
Editorial
}

\section{Non-Invasive Assessment of Myocardial Oxygenation with MRI}

\section{Jie Zheng}

Mallinckrodt Institute of Radiology, Washington University in St. Louis, St. Louis, Missouri 63110, USA

Cardiovascular disease (CVD) remains the number one cause of death in most western countries, and a major cause of hospital admissions. One major disorder of CVD is myocardial ischemia, resulting from upstream coronary artery stenosis or impaired microcirculation. The common clinical manifestations of myocardial ischemia are angina (stable and unstable), myocardial infarction (heart attack), heart failure, arrhythmia (irregular heart beat), and sudden death. The cause of myocardial ischemia is the lack of oxygen-rich blood supply to the myocardial tissue. Balance of myocardial oxygen supply and demand is essential for maintaining the integrity of myocardial metabolism and mechanical function. Strictly speaking, myocardial ischemia is defined by the imbalance of oxygen supply and demand in the myocardial tissue. The assessment of myocardial blood flow is not always adequate to determine whether the tissue is ischemic or not.

Conversely, non-invasive assessment of this balance will have a significant clinical impact on the diagnosis and monitoring of cardiac patients with ischemia. Positron emission tomography (PET) has long been recognized as the gold standard for quantifying myocardial oxygenation in terms of myocardial oxygen consumption rate $\left(\mathrm{MVO}_{2}\right)$ using a

*Address correspondence to this author at the Mallinckrodt Institute of Radiology, Washington University in St. Louis, St. Louis, Missouri 63110, USA; Tel: 314-747-4608; Fax: 314-747-3882;

E-mail: zhengj@mir.wustl.edu radioactive tracer (e.g., ${ }^{11} \mathrm{C}$-Acetate or ${ }^{15} \mathrm{O}$-oxygen). However, its low spatial resolution, exposure to radiation, and relatively high costs have limited the widespread use of this imaging modality. Cardiac magnetic resonance (CMR) has been increasingly recognized as an important non-invasive cardiac imaging modality, with high spatial resolution, excellent soft-tissue contrast, and lack of radiation. Myocardial BOLD imaging (Blood Oxygen Level Dependent) is considered one potential candidate for the assessment of myocardial oxygenation. Currently, efforts have been made to develop a variety of both qualitative and quantitative imaging techniques. Many reports in animal and human studies have clearly demonstrated the feasibility of this method.

In this mini-hot topic issue, CMR techniques to address cardiac BOLD imaging are comprehensively reviewed with a balance between technical development and clinical applications. In the contribution entitled, "BOLD myocardial imaging: Advances for clinical and physiological applications", Peter S et al., provided a concise review on the history and status of research using cardiac BOLD imaging for the detection of abnormality in myocardial oxygenation, particularly in the use of myocardial $\mathrm{T}_{2} *$ approach. A global disease model, hypertensive hypertrophy, was assessed by Beache et al., showing a detectable reduction in myocardial $\mathrm{T}_{2}{ }^{*}$. A more detailed review of the myocardial $\mathrm{T}_{2}$ approach 
is highlighted in the contribution entitled, "Myocardial BOLD Imaging with $T_{2}$ relaxation", authored by Ghugre NR and Wright GA. This group, led by Dr. Wright, is a highly respected MR research laboratory. The $\mathrm{T}_{2}$ approach represents the success of the investigators in the development and promotion of quantitative evaluation of tissue oxygenation, from skeletal muscle to myocardial tissue, even to the whole body oxygenation. To pursue a clinically friendly and robust measurement method, Dharmakumar and Li et al., have systematically developed and evaluated an oxygen-sensitive balanced steady-state free precession (SSFP) imaging approach. The validation study in animals with a variety of changes in myocardial oxygenation demonstrated convincing evidence that this technique is robust against many image artifacts, with good signal-to-noise ratio and sensitivity to changes in myocardial oxygenation. In addition, 3T BOLD imaging appears to provide better sensitivity than $1.5 \mathrm{~T}$. Until now, myocardial BOLD imaging method is increasingly used in the diagnosis of patients with coronary artery disease. Lastly, Muccigrosso et al. explained the importance of determination of regional myocardial oxygenation, particularly including that of sub-endocardium. Specific pathology indices, such as myocardial oxygenation extraction fraction and $\mathrm{MVO}_{2}$, can be readily measured during pharmacologically induced hyperemia. A new method to directly assess myocardial oxygenation, with a ${ }^{17} \mathrm{O}$-labelled tracer agent, is now in the development phase.

In summary, there is a compelling body of evidence for the role and feasibility of CMR oxygenation imaging for both basic science research and clinical diagnosis. These emerging new techniques open new opportunities to increase our understanding of the oxidative metabolic events involved in the progression of ischemia and may help the development of novel therapeutical treatments for CVD patients with myocardial ischemia.

\section{(C) Jie Zheng; Licensee Bentham Open.}

This is an open access article licensed under the terms of the Creative Commons Attribution Non-Commercial License (http://creativecommons.org/licenses/by$\mathrm{nc} / 3.0 /$ ), which permits unrestricted, non-commercial use, distribution and reproduction in any medium, provided the work is properly cited. 